\title{
Characterization of Silica Particles and Concentration Dependent Adsorption Study of Biomolecules
}

\author{
M. A. Rahman, M. A. J. Miah, H. Ahmad* \\ Department of Chemistry, University of Rajshahi, Rajshahi 6205, Bangladesh
}

\begin{abstract}
Submicron-sized silica particles were initially characterized by scanning electron micrograph, FTIR spectroscopy and XRD analysis. The observations revealed that the highly dispersed amorphous silica particles were relatively monodispersed and the particles surface was covered with hydrogen bonded $\mathrm{OH}$ groups. Relatively higher magnitude of adsorption of cationic emulsifier and coagulation of silica particles suggested that particles were negatively charged. A comparative study of adsorption behavior of different biomolecules such as trypsin, albumin and lysozyme was also done at the respective isoelectric $\mathrm{pH}$ value. The magnitude of adsorption increased with the increase in the amount of immobilization per unit mass of silica particles.
\end{abstract}

Key words: Silica, Amorphous, Adsorption behavior, Emulsifier, Biomolecules

\section{Introduction}

Monodispersed colloidal particles of any size and shape have become the most significant area of research considering the large surface area and ease to control the surface property. Depending on the surface properties, these particles may find applications in textiles, electronics, chromatography, coating and biotechnology (Ahmad et al. 2003, Kang et al. 2006, Lin et al. 2006, Li et al. 2007, Norde 1997, Yang et al. 2005). The surface properties also play an important role in determining the colloidal behavior of the latex.

In this investigation submicron-sized silica particles obtained from Nippon Shokubai Co. Ltd. Japan has been taken into account for surface characterization. The adsorption of proteins and emulsifier molecules on the solid surfaces is known to depend on general physicochemical surface properties such as hydrophobicity and surface charge density. Hydrophobic interaction has been found to play an important role in protein adsorption. Krisdhasima et al. (1992) studied the adsorption of $\beta$-lactogobulin on chemically modified silicon surfaces and found that the increase in hydrophobicity of the surface increased the amount of adsorption. Several articles (H. Ahmad et al. 2004, 2005, 2006, 2007 and 2008) reported similar results in the magnitude of adsorption for different biomolecules on organic polymer latexes. Zoungrana et al (1997) observed that $\alpha$ chemotropism and cutinase adsorbed on hydrophilic silica surface retained their native conformation. Vertegel et al (2004) studied the adsorption of chicken egg albumin on sil ica nanoparticles of various diameters. The adsorption patterns, protein structure and function were strongly dependent on the size of nanoparticles. So far most of the adsorption studies done on silica particles dealt with one type of protein and the silica particles used were not spectroscopically analyzed. The adsorption behaviors of different cationic and anionic surfactants have also been studied in details (Lindman et al. 2004, Thibaut et al. 2000). Despite the considerable researches dealing with silica particles, it is well known that such adsorption kinetics depending on surface properties is variable with processing condition, dispersity index, size and shape of the particles. Considering this aspect, silica particles obtained from Nippon Shokubai Co. Ltd. Japan have been studied for surface characterization. The modification of such silica particles with organic polymer would be attempted in the next article.

\section{Materials and method}

Silica dispersion donated by Nippon Shokubai Co. Ltd. Japan, was used after centrifugal washing. Styrene of monomer grade (Fluka, Chemika, Switzerland) was distilled under reduced pressure. Poly(vinyl pyrrolidone) (PVP) from LOBA chem. India, and tricaprylylmethyl ammonium chloride (aliquate ${ }^{336}$ ) from Fluka, Chemika, Switzerland were used as received. Sodium dodecyl sulfate (SDS) and hexadecyl trimethyl ammonium bromide (HTABr) from Fluka Chemika, Switzerland were used as anionic and cationic

\footnotetext{
* Corresponding author: E-mail: : samarhass@yahoo.com
} 
emulsifiers. The biomolecules used were trypsin (TR) from E. Merck, Germany; albumin (AL) from LOBA Chem., India and lysozyme (LZ) from Fluka, Biochemika, Switzerland. Other chemicals used were of reagent grade. Distilled deionized water of conductivity less than $5 \mu \mathrm{S} / \mathrm{cm}$ was used for all the experiments.

Scanning electron microscope (SEM) (LEO Electron Microscopy Ltd, UK) was used to see the images of PS particles. Helios Gamma Single-beam UV-Visible Spectrophotometer from Unicam, UK and FTIR-8900, Japan were used for the analyses.

\section{Preparation of polystyrene (PS) particles by dispersion polymerization}

PS particles were prepared by dispersion polymerization of $40 \mathrm{~g}$ styrene dissolving in a mixture of $200 \mathrm{~mL}$ ethanol and $10 \mathrm{~mL}$ water in presence of AIBN (0.6 g) as oil soluble initiator. PVP (1.6 g) and aliquate ${ }^{336}$ (0.46 g) were used as stabilizer and co-stabilizer respectively. Polymerization was carried out in a three necked round bottomed flask under nitrogen atmosphere at $70^{\circ} \mathrm{C}$ for $12 \mathrm{~h}$ with stirring rate maintained at $80 \mathrm{rpm}$. Conversion of styrene to PS was measured gravimetrically and it was nearly $100 \%$.

\section{Adsorption of biomolecules}

A mixture of $20 \mathrm{~mL}$ was prepared from purified silica dispersion (solid $0.1 \mathrm{~g}$ ) and biomolecules of varying concentrations. In each measurement, for $0.1 \mathrm{~g}$ of silica particles $2 \mathrm{mg}$, $5 \mathrm{mg}, 10 \mathrm{mg}, 15 \mathrm{mg}, 20 \mathrm{mg}$ and $30 \mathrm{mg}$ of biomolecules were used, respectively. The $\mathrm{pH}$ value of the mixture was immediately adjusted with phosphate buffer at the respective isoelectric point (TR, pH 10; AL, pH 6; LZ, pH 10.5). The mixture was allowed to stand at $30^{\circ} \mathrm{C}$ for $45 \mathrm{~min}$, and then centrifuged at 7,000 rpm for 15 minutes. In order to remove the dust particles completely, the supernatant was centrifuged once more at 7,000 rpm. The concentration of the biomolecule in the supernatant was determined by UV-visible spectrophotometer at $280 \mathrm{~nm}$. The magnitude of adsorption was calculated by subtracting concentration of biomolecule in the medium from that of the initial concentration. Calibration curve was used for this purpose. The adsorption of TR on PS particles was also measured under similar conditions.

\section{Adsorption of emulsifiers}

For each measurement, a mixture of $30 \mathrm{~mL}$ was prepared from purified silica dispersion (solid, $0.1 \mathrm{~g}$ ) and emulsifier (SDS/HTABr) aqueous solution. The concentration of the emulsifier in the mixture was kept below the critical micelle concentration to avoid the formation of micelle. The $\mathrm{pH}$ of mixture was immediately adjusted to 7.0 using dilute $\mathrm{KOH}$ solution. The dispersion-emulsifier mixture was then allowed to stand for $2 \mathrm{~h}$ at $30^{\circ} \mathrm{C}$. In order to examine the adsorption behavior of the emulsifier onto the composite particles the conductance was recorded at $30^{\circ} \mathrm{C}$. The magnitude of emulsifier adsorbed was calculated by subtracting the emulsifier concentration in the medium from the initial concentration. The emulsifier concentration was obtained using calibration curves, representing the relationship between the concentration and the conductance, for emulsifier aqueous solution at the $\mathrm{pH}$ value of 7.0, assuming that the adsorbed emulsifier molecules don't contribute to the conductance.

\section{Results and Discussion:}

Fig. 1 shows the SEM photographs of silica particles. The particles are almost spherical. The average diameter and coefficient of variation are $0.52 \mu \mathrm{m}$ and $3.52 \%$, indicating that particles are relatively monodispersed.

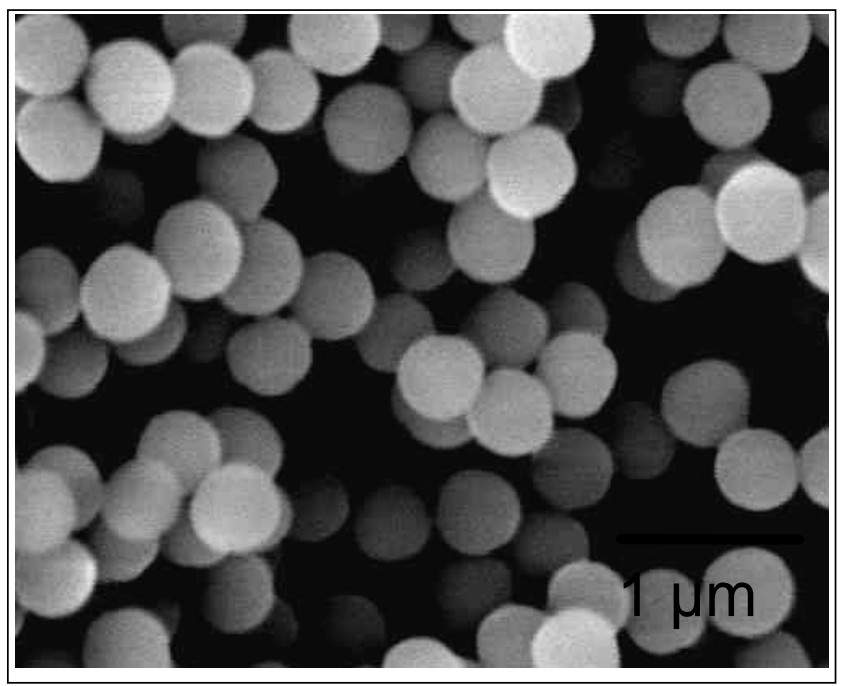

Fig. 1. SEM photograph of silica particles.

FTIR spectrum of silica particles is shown in Fig. 2. The signal at $1083.9 \mathrm{~cm}^{-1}$ is attributed to Si-O-Si stretching vibration in $\mathrm{SiO}_{4}$ units and that at $472 \mathrm{~cm}^{-1}$ is attributed to Si-OSi bending. The peak at $800.4 \mathrm{~cm}^{-1}$ is assigned to Si-O-Si bond vibration between two adjacent tetrahedra (Ohtsuki et al. 1992, Kim et al. 1989), occurred in silica gel (Nyquist et al. 1991). The signal appeared at $954.7 \mathrm{~cm}^{-1}$ is due to $\mathrm{Si}-\mathrm{O}$ terminal non-bridging vibration (Costantini et al. 2006). The broad signal appeared at $3421 \mathrm{~cm}^{-1}$ can be assigned to bonded $\mathrm{OH}$ groups on the surface of silica particles

The XRD pattern of silica particles shown in Fig. 3 exhibits a broad reflection centered at $2 \theta=22^{\circ}$. This broad reflection band suggests that silica particles are substantially amorphous in character. 


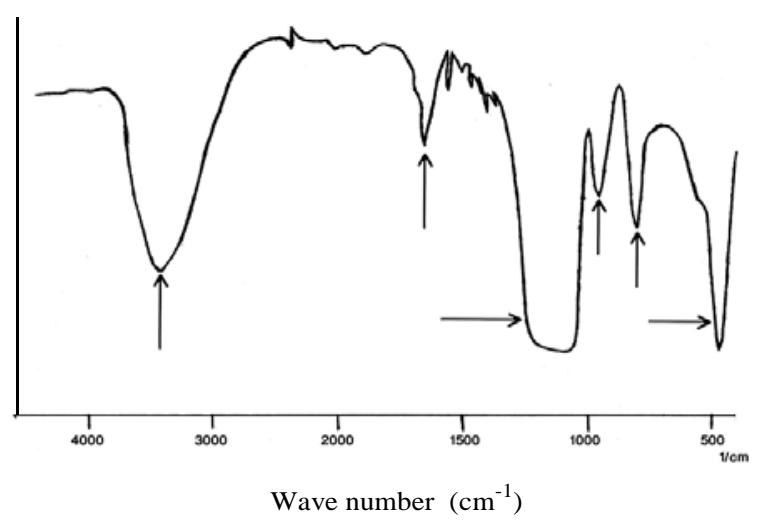

Fig. 2. FTIR spectrum of washed silica particles.

The variations in magnitude of TR, AL and LZ adsorbed on silica particles in presence of different amounts of biomolecules in silica-biomolecule dispersion are shown in Fig. 4. The measurements were carried out at isoelectric point of the respective biomolecule to eliminate the effect of ionic interaction. In each case the magnitude of adsorption increased with the increase in the amount of immobilization and reached almost plateau level beyond $200 \mathrm{mg}$ of biomolecules. The adsorbed biomolecules at the plateau level is

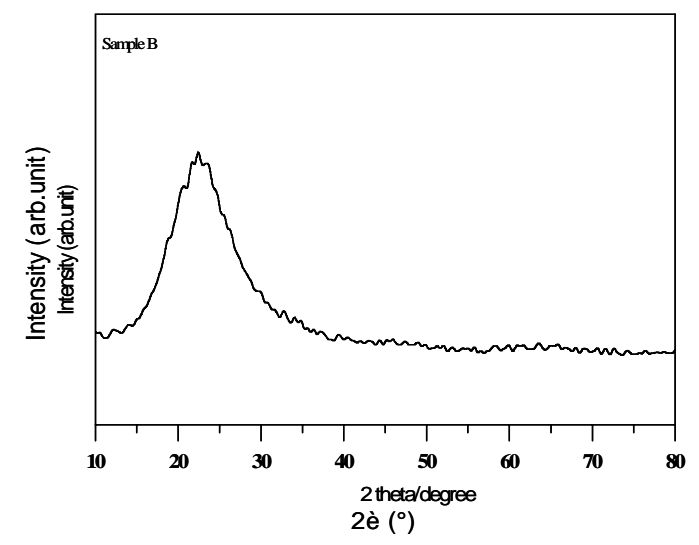

Fig. 3. X-ray diffraction spectra of washed silica particles.

expected to have monolayer coverage on the particles surface. The magnitude of adsorption is influenced by various factors such as properties of proteins, the solid substrate surface and environmental conditions. In this study the solid substrate and environment condition remained the same. The difference in the magnitude of adsorption among different biomolecules is therefore attributed to the difference in properties such as size, flexibility, amino acid composition and steric conformation. The magnitude of adsorption on the hydrophilic surface is usually lower unless there is electrostatic attraction than on the hydrophobic surface. Fig. 5 shows that the magnitude of adsorption of TR on polystyrene (PS) particles is much higher than that on silica particles measured under identical conditions with varying concentra- tion of TR. This suggests that the surface of silica particles is relatively hydrophilic and is covered with bonded $\mathrm{OH}$ groups, which have also been supported by FTIR spectra as shown in Fig. 2.

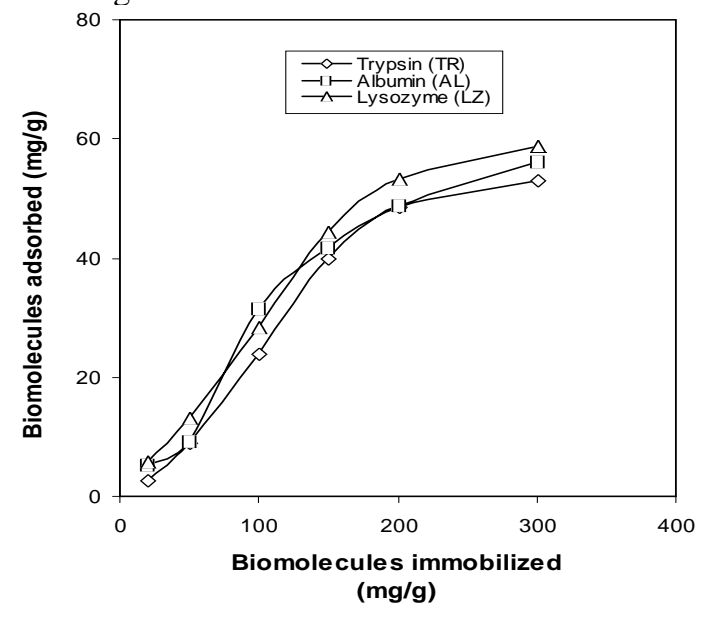

Fig. 4. Effect of concentration of biomolecules in silicabiomolecule dispersion on the magnitude of adsorption at $30^{\circ} \mathrm{C}$ measured at the respective isoelectric point ((TR, pH 10; AL, pH 6; LZ, pH 10.5).

The magnitude of adsorption for anionic SDS and cationic $\mathrm{HTABr}$ emulsifiers were also measured on silica particles under similar conditions. The magnitude of adsorption of cationic HTABr (21.94 mg/g of particles) was higher than that of anionic SDS (4 mg/g of particles). Moreover, contrary to the anionic emulsifier, the silica particles were coagulated in presence of cationic emulsifier. This behavior indicates that silica dispersion is stabilized due to the presence of negative charges on the particles surface and addition of cationic emulsifier neutralized the negative charges which ultimately produced coagulation.

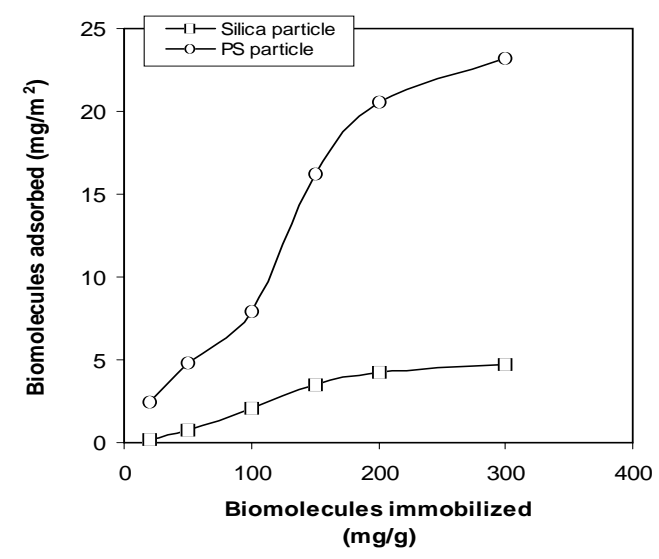

Fig. 5. Magnitude of TR adsorbed on polystyrene (PS) and silica particles measured under identical conditions. pH: 10 ; temperature: $30^{\circ} \mathrm{C}$; particles: 0.1 g. TR: variable. 
The above study revealed that silica particles are hydrophilic, anionically charged and mostly amorphous in character. These particles can be used in biomedical field or can be modified/ functionalized to diversify the application in biotechnology.

\section{Acknowledgements}

Authors would like to thank Nippon Shokubai Co. Ltd. for providing the silica dispersion as a free sample. Thank is also to Md. Abdul Mannan, a PhD student at Saga University, Japan for XRD analysis.

\section{References}

Ahmad H., Miah M. A. J. and Rahman M. M. (2003). Preparation of micron-sized composite polymer particles containing hydrophilic 2-hydroxyethyl methacrylate and their biomedical applications, Colloid Polym. Sci., 281: 988.

Ahmad H., Khatun M. A. and Miah M. A. J. (2004). A comparative study of the adsorption behavior of biomolecules on PMMA/P(MMA-AAm) and PS/ P(MMAAAm) composite polymer particles, J. Bang. Chem. Soc., 17(2): 153.

Ahmad H., Rahman M. M. and Miah M. A. J. (2005) Preparation of micron-sized epoxy functionalized polystyrene particles and adsorption of biomolecules, $J$. Polym. Mater., 21: 27.

Ahmad H., Hossain M. A., Islam A. H. M. T. and Miah M. A. J. (2006). Preparation kinetics of monosized cyano functionalized copolymer particles by dispersion copolymerization and adsorption behavior of biomolecules, ACGC Chem. Res. Comm., 20: 24.

Ahmad H., Hossain M. M., Rahman M. M. and Miah M. A. J. (2007). Monodispersed carboxylated composite polymer microspheres and physical immobilization of biomolecules, Polym. J., 39: 428.

Ahmad H., Hossain M. E., Rahman M. A., Rahman M. M. and Miah M. A. J. (2008). Carboxyl functionalized poly(methyl methacrylate-acrylic acid-ethylene glycol dimethacrylate) copolymer particles and their amination with amine nucleophiles, e-Polymers, no. 096.

Costantini A., Luciani G., Annunziata G.., Silvestri B. and Branda F. (2006). Swelling properties and bioactivity of silica gel/pHEMA nanocomposites, J. Mater. Sci: Mater. Med. 17: 319
Kang K., Kan C. and Yeung A. Liu. D. (2006). The immobilization of trypsin on soap-free P(MMA-EA-AA) latex particles, Materials Sci. Eng., 26:664.

Kim C. Y., Clark A. E. and Hench L. L. (1989). Early stages of calcium-phosphate layer formation in bioglasses, $J$. Non-cryst. Solids, 113: 195

Krisdhasima V., McGuire J. and Sproull R. (1992). Surface hydrophobic influence on ?-lactoglobulin adsorption kinetics, J. Colloid Interface Sci., 154: 337.

Lin C. L. and Chiu W. Y. (2006). Polypyrrole/Poly(N-isopropylacrylamide-co-acrylic acid) thermosensitive and electrically conductive composite microgels, J. Polym. Sci. Polym. Chem. Ed., 44: 1648.

Li G.., Song Y., Yang X. and Huang W. (2007). Preparation of poly (divinylbenzene-co- $\mathrm{N}$-isopropylacrylamide) microspheres and their hydrogen-bonding assembly behavior for raspberry-like core-corona polymer composite, J. App. Polym. Sci., 104: 1350.

Nyquist R. A. and Kagel R. O. (1991). Infrared spectra of inorganic compounds (Academic Press Inc., San Diego, California)

Ohtsuki C., Kokubo T. and Yamamuro T. (1992). Mechanism of apatite formation on $\mathrm{Cao}-\mathrm{SiO}_{2}-\mathrm{P}_{2} \mathrm{O}_{5}$ glasses in a simulated body fluid, J. Non-cryst. Solids, 143: 84.

Terada E., Samoshina Y., Nylander T. and Lindman B. (2004). Adsorption of cationc cellulose derivatives/anionic surfactant complexes onto solid surfaces. I. silica surfaces, Langmuir, 20: 1753.

Thibaut A., Misselyn-Bauduin A. M., Borze G. and. Jérôme R. (2000). Adsorption of poly(vinylpyrrolidone)/surfactant(s) mixtures at the silica/water interface: a calorimetric investigation, Langmuir, 16: 9841.

Vertegel A. A., Siegel R. W. and Dorkick J. S. (2004). Silica nanoparticle size influences the structure and enzymatic activity of adsorbed lysozyme, Langmuir, 20: 6800.

Yang M. and Dan Y. (2005). Preparation and characterization of poly(methyl methacrylate)/titanium oxide composite particles, Colloid Polym. Sci., 284: 243.

Zoungrana T., Findenegg G. H. and Norde, W. (1997). Structure, stability, and activity of adsorbed enzymes, J. Colloid Interface Sci., 190: 437.

Received : September 23, 2008;

Accepted : March 16, 2010 